\title{
An Extended Analytic Solution of Combined Refraction and Diffraction of Long Waves Propagating over Circular Island
}

\author{
Yu-Shu Kuo, ${ }^{1}$ Tai-Wen Hsu, ${ }^{1,2}$ \\ Chia-Cheng Tsai, ${ }^{3}$ and Yu-Hsuan Huang ${ }^{1}$ \\ ${ }^{1}$ Department of Hydraulic and Ocean Engineering, National Cheng Kung University, Tainan 701, Taiwan \\ ${ }^{2}$ Research Center of Ocean Energy and Strategy, National Taiwan Ocean University, Keelung 202, Taiwan \\ ${ }^{3}$ Department of Marine Environmental Engineering, National Kaohsiung Marine University, \\ Kaohsiung 811, Taiwan
}

Correspondence should be addressed to Chia-Cheng Tsai, tsaichiacheng@mail.nkmu.edu.tw

Received 18 June 2012; Revised 5 September 2012; Accepted 24 September 2012

Academic Editor: Michael Meylan

Copyright (C) 2012 Yu-Shu Kuo et al. This is an open access article distributed under the Creative Commons Attribution License, which permits unrestricted use, distribution, and reproduction in any medium, provided the original work is properly cited.

\begin{abstract}
An analytic solution of long waves scattering by a cylindrical island mounted on a permeable circular shoal was obtained by solving the linear long wave equation (LWE). The solution is in terms of the Bessel function expressed by complex variables. The present solution is suitable for arbitrary bottom configurations described by a power function with two independent parameters. For the case of the paraboloidal shoal, there exists a singular point $(\alpha=2)$ which can be removed using Frobenius series, where $\alpha$ is a real constant. The present solution is reduced to Yu and Zhang's (2003) solution for impermeable circular shoal. The numerical results show some special features of the combined effect of wave refraction and diffraction caused by a porous circular island. The effect of key parameters of the island dimension, the shoal slope, and permeability on wave scattering was discussed based on the analytic solution.
\end{abstract}

\section{Introduction}

The combined effect of wave refraction and diffraction is caused by the varying bottom topography or the presence of islands and structures. The mild-slope equation (MSE) was first derived by Berkhoff [1] and then was extended by Porter and Staziker [2], Hsu and Wen [3] to the rapidly varying sea bottom configuration. Up to now, the MSE still plays an important role in coastal and ocean engineering practice, as it can be widely implemented to solve the problems of combined refraction and diffraction based on linear wave theory.

Although numerical models are commonly used in practical applications, it is still limited by the problems of accuracy and numerical stability. Numerical results are often 
needed to be verified by comparing with measured data or analytic solutions. In some cases, it takes time and expensive cost for laboratory experiments because of the complexity in completion of the model and facilities setup. An alternate is to look for analytic solution that could provide more clear expressions in which the physical properties of wave motion can be used to examine the predictability and validity of the numerical models.

A typical example of the analytic study of combined refraction and diffraction is the case when the long-wave approximation is applied to the MSE in one dimension or axisymmetric bathymetry in two dimensions. Homma [4] is a pioneer to obtain the solution of long-wave equation (LWE) by considering long waves propagating over the top of a parabolic shoal. The solution was further extended and applied by Vastano and Reid [5] and Jonsson et al. [6]. Different geometries of circular or conical island were explored by Fujima and Goto [7], Fujima et al. [8], Zhang and Zhu [9], and Zhu and Zhang [10]. The research field is still under rapid developments. Details can be referred to recent articles of Jung et al. [11], Niu and Yu [12], Liu and Xie [13], and Jung and Lee [14]. Furthermore, there are also studies, such as Liu et al. [15] and Niu and Yu [16], using Hunt's [17] approximate solution of the implicit dispersion equation to solve the MSE without the long-wave approximation.

The present investigation is to extend Yu and Zhang's [18] analytic solution to long wave propagation over a porous circular island with arbitrary topography. The combined effect of refraction and diffraction of long wave motion as well as key features of the intensity of wave ray focus behind the shoal is presented. A general solution was derived based on the mild slope equation (MSE). The wave motion with combined refraction and diffraction was described by the Bessel function $(\alpha \neq 2)$ and Frobenius series. The effect of key parameters on wave scattering is intensively discussed based on the analytic solution.

\section{Theoretical Formulation}

The long wave equation for combined refraction and diffraction of waves propagating over porous media is express as

$$
\nabla_{h}\left(\left(h_{w}+\delta h_{p}\right) \nabla_{h} \phi\right)+k^{2}\left(h_{w}+\delta h_{p}\right) \phi=0
$$

where $\phi$ is the velocity potential, $\nabla_{h}=(\partial / \partial x, \partial / \partial y)$ the horizontal gradient operator, $h_{w}$ the thickness of the water, $h_{p}$ the thickness of porous layer, $k$ is the wavenumber and $\delta$ is defined as $n_{0} /\left(S+\mathbf{i} f_{p}\right)$ with $n_{0}$ the porosity, $S=n_{0}+\left(1-n_{0}\right) C_{M}$, the inertial coefficient, $C_{M}$ the virtual mass coefficient, $\mathbf{i}=\sqrt{-1}$ the unit complex number, and $f_{p}$ the friction coefficient. In the derivation of (2.1), the time harmonic $e^{\mathrm{i} \omega t}$ is assumed with $\omega$ being the angular frequency of the incident wave. Here, the angular frequency $\omega$ is related to the wavenumber $k$ by the following dispersion relation:

$$
\frac{\omega^{2}}{g}=k\left(\delta k h_{p}+k h_{w}\right)
$$

Equations (2.1) and (2.2) can be derived from the mild-slope equation of Rojanakamthorn et al. [19] by neglecting terms of orders $O\left(k^{2} h_{w}^{2}, k^{2} h_{p}^{2}, k^{2} h_{w} h_{p}\right)$. Note that (2.1) and (2.2) are only valid for long waves, that is, the relative depth is restricted in the shallow water region, 
that is, $h_{w} / L \leq 1 / 20$ and $h_{p} / L \leq 1 / 20$ where $L$ is the wave length. More details can be found in the Appendix.

In order to study the wave scattering over a circular porous shoal, we assume that the shoal is symmetric and of the following form:

$$
\begin{gathered}
h_{w}=h_{w}(r)=\frac{\bar{h}_{w}}{r_{0}^{\alpha}} r^{\alpha} \alpha r^{\alpha}, \quad r \leq r_{0}, \\
h_{p}=h_{p}(r)=\frac{\bar{h}_{p}}{r_{0}^{\alpha}} r^{\alpha} \alpha r^{\alpha}, \quad r \leq r_{0},
\end{gathered}
$$

where $r$ is the distance of any point on the shoal from the center, $r_{1}$ is the radius of the circular island, $r_{0}$ is the radius of the impermeable circular shoal, $\bar{h}_{w}$ and $\bar{h}_{p}$ are the depths of water and porous layers, respectively, for $r>r_{0}$ and $\alpha$ is the power parameter of bottom shape. The definition sketch of the permeable circular island is presented in Figure 1.

Using the method of separation variables, the velocity potential is written as

$$
\phi=\sum_{n=0}^{\infty} R_{n}(r) \Theta_{n}(\theta)
$$

in which $\Theta_{n}$ is the eigenfunction given by

$$
\Theta_{n}(\theta)=C_{1 n} \cos n \theta+C_{2 n} \sin n \theta \quad(n=0,1,2, \ldots),
$$

where $C_{1 n}$ and $C_{2 n}$ are coefficients to be determined. $C_{2 n}$ is set to be zero due to the symmetric property of the present problem with respect to the coordinate $x$. For convenience of computational procedure, the coefficient $C_{1 n}$ is specified as $C_{1 n}=1$. Substituting (2.4) and (2.5) into (2.1) gives

$$
r^{2} \frac{d^{2} R_{n}}{d r^{2}}+r\left(1+\frac{r}{\widetilde{h}} \frac{d \tilde{h}}{d r}\right)+\left(\frac{\omega^{2} r^{2}}{g \tilde{h}}-n^{2}\right) R_{n}=0
$$

where $\tilde{h}$ is an equivalent water depth defined by

$$
\tilde{h}=h_{w}+\delta h_{p}
$$

The following dimensionless variables are introduced for convenience of mathematical formulation:

$$
\rho=\frac{\omega^{2} r}{g}, \quad \xi_{w}^{2}=\frac{\omega^{2} h_{w}}{g}, \quad \xi_{p}^{2}=\frac{\omega^{2} h_{p}}{g}
$$



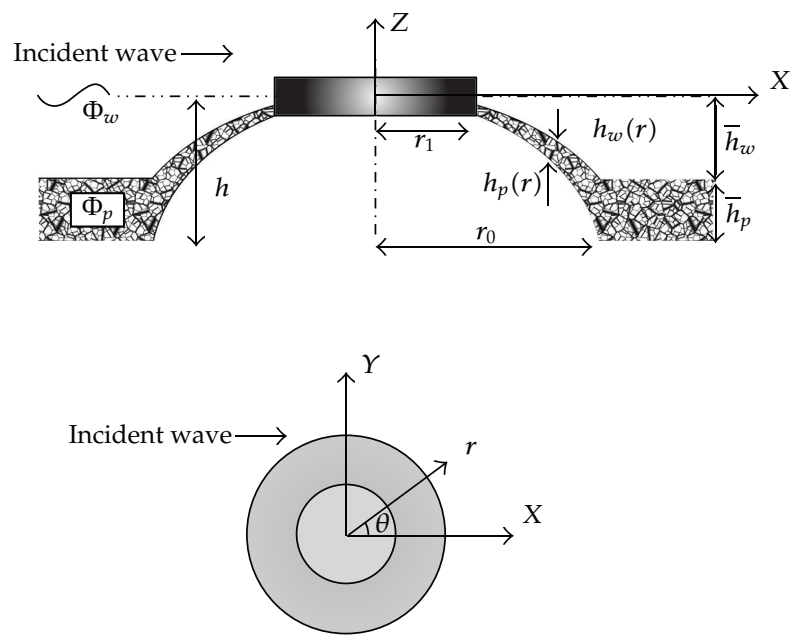

Figure 1: Definition sketch of a circular island.

Substitution of $\xi_{w}^{2}$ and $\xi_{p}^{2}$ from (2.8) into (2.3) leads to the resultant equations.

$$
\begin{aligned}
& \xi_{w}^{2}=\frac{\bar{\xi}_{w}^{2}}{\rho_{0}^{\alpha}} \rho^{\alpha}, \quad \rho \leq \rho_{0}, \\
& \xi_{p}^{2}=\frac{\bar{\xi}_{p}^{2}}{\rho_{0}^{\alpha}} \rho^{\alpha}, \quad \rho \leq \rho_{0} .
\end{aligned}
$$

Equation (2.10) is multiplied by $\delta$ and added with (2.9). The following equation is thus obtained, that is

$$
\xi^{2}=\xi_{w}^{2}+\delta \xi_{p}^{2}=\frac{\bar{\xi}_{w}^{2}}{\rho_{0}^{\alpha}} \rho^{\alpha}+\delta \frac{\bar{\xi}_{p}^{2}}{\rho_{0}^{\alpha}} \rho^{\alpha}=\left(\frac{\bar{\xi}_{w}^{2}}{\rho_{0}^{\alpha}}+\delta \frac{\bar{\xi}_{p}^{2}}{\rho_{0}^{\alpha}}\right) \rho^{\alpha}=\frac{1}{\beta^{2}} \rho^{\alpha} .
$$

And, (2.6) is rearranged with the help of the dimensionless variable $\rho=\omega^{2} r / g(2.8)$ and the result reads

$$
\rho^{2} \frac{d^{2} R_{n}}{d \rho^{2}}+(\alpha+1) \rho \frac{d R_{n}}{d \rho}+\left(\beta^{2} \rho^{2-\alpha}-n^{2}\right) R_{n}=0
$$

For the special case of $\alpha=2,(2.12)$ is solved by making use of the Frobenius series, the solution is given by

$$
R_{n}= \begin{cases}C_{3 n} \rho^{-1+\mu_{n}}+C_{4 n} \rho^{-1-\mu_{n}} & \text { if } 1+n^{2}-\beta^{2} \neq 0 \\ C_{3 n} \rho^{-1}+C_{4 n} \rho^{-1} \ln \rho & \text { if } 1+n^{2}-\beta^{2}=0\end{cases}
$$


where $C_{3 n}$ and $C_{4 n}$ are constants, and $\mu_{n}$ is represented by

$$
\mu_{n}=\sqrt{\left|1+n^{2}-\beta^{2}\right|}
$$

We introduce two dimensionless parameters $Q_{n}=\rho^{\alpha / 2} R_{n}$ and $\chi=(2 \beta /|2-\alpha|) \rho^{1-\alpha / 2}$ for $\alpha \neq 2$, (2.6) is rewritten in the form

$$
x^{2} \frac{d^{2} Q_{n}}{d x^{2}}+x \frac{d Q_{n}}{d x}+\left(x^{2}-v_{n}^{2}\right) Q_{n}=0
$$

where $v_{n}=\sqrt{\left(\alpha^{2}+4 n^{2}\right) /(|2-\alpha|)}$.

The general solution of (2.15) is the Bessel function expressed as

$$
Q_{n}=C_{3 n} J_{v_{n}}(X)+C_{4 n} Y_{v_{n}}(X) \quad(n=0,1,2 \ldots)
$$

where $J_{v_{n}}$ and $Y_{v_{n}}$ are the Bessel functions of the first and second kind of $v_{n}$ order. Substitution the corresponding boundary conditions in (2.16) results in the particular solution for $\alpha \neq 2$ given by

$$
R_{n}(\rho)=\rho^{-\alpha / 2}\left[C_{3 n} J_{v_{n}}\left(\frac{2 \beta}{|2-\alpha|} \rho^{1-(\alpha / 2)}\right)+C_{4 n} Y_{v_{n}}\left(\frac{2 \beta}{|2-\alpha|} \rho^{1-(\alpha / 2)}\right)\right] \quad(n=0,1,2, \ldots) .
$$

For an arbitrary bathymetry of a circular island, the solution is thus written as follows

$$
R_{n}(\rho)=C_{3 n} R_{1 n}(\rho)+C_{4 n} R_{2 n}(\rho)
$$

where

$$
\begin{aligned}
& R_{1 n}(\rho)= \begin{cases}\rho^{-1+\mu_{n}} & \text { if } \alpha=2 \& 1+n^{2}-\beta^{2} \neq 0, \\
\rho^{-1} & \text { if } \alpha=2 \& 1+n^{2}-\beta^{2}=0, \\
\rho^{-\alpha / 2} J_{v_{n}}\left(\frac{2 \beta}{|2-\alpha|} \rho^{1-(\alpha / 2)}\right) & \text { if } \alpha \neq 2,\end{cases} \\
& R_{2 n}(\rho)= \begin{cases}\rho^{-1-\mu_{n}} & \text { if } \alpha=2 \& 1+n^{2}-\beta^{2} \neq 0, \\
\rho^{-1} \ln \rho & \text { if } \alpha=2 \& 1+n^{2}-\beta^{2}=0, \\
\rho^{-\alpha / 2} Y_{v_{n}}\left(\frac{2 \beta}{|2-\alpha|} \rho^{1-(\alpha / 2)}\right) & \text { if } \alpha \neq 2 .\end{cases}
\end{aligned}
$$

It is noted that the LWE of (2.1) has been solved for long waves propagating over a permeable circular island. The velocity potential is finally expressed as

$$
\phi=\sum_{n=0}^{\infty}\left[C_{3 n} R_{1 n}(\rho)+C_{4 n} R_{2 n}(\rho)\right] \cos n \theta .
$$


Considering an incident wave expressed in a nondimensional form:

$$
\phi_{0}=A_{I} e^{\mathrm{i} k_{0} x}=A_{I} \sum_{n=0}^{\infty} \mathbf{i}^{n} \varepsilon_{n} J_{n}\left(k_{0} r\right) \cos n \theta=A_{I} \sum_{n=0}^{\infty} \mathbf{i}^{n} \varepsilon_{n} J_{n}\left(\kappa_{0} \rho\right) \cos n \theta
$$

where $A_{I}$ is the reference wave height defined as $A_{I}=\phi_{0}(\rho=0)$. In addition, the wavenumber $k_{0}$ for $r>r_{0}$ can be obtained from the dispersion relation (2.2), and the dimensionless wave number $\kappa_{0}$ is defined as $k_{0} g / \omega^{2}$. In addition, $\varepsilon_{n}$ is the Jacobian parameter given by

$$
\varepsilon_{n}= \begin{cases}1 & n=0 \\ 2 & n \geq 1\end{cases}
$$

The physical problem is presented in Figure 1. Following Yu and Zhang [18], the method of eigenexpansion is adopted to solve the problem. The study domain is divided into two subregions on the horizontal plane: the finite region with varying depth $\left(r_{1}<r<r_{0}\right)$ and semi-infinite far region with a porous constant depth $\left(r>r_{0}\right)$. The general solution of the complex velocity potential subject to the Somerfield condition as well as the symmetric condition is written in the form

$$
\begin{gathered}
\phi_{1}=\phi_{0}+\sum_{n=0}^{\infty} D_{n} \Psi_{n}(\rho), \\
\Psi_{n}(\rho) \equiv H_{n}\left(\kappa_{0} \rho\right),
\end{gathered}
$$

where $H_{n}$ is the Hankel function of the first kind of the $n$th order, $D_{n}$ are complex coefficients to be determined.

In the finite region, the permeable bottom configuration is represented by (2.3). The complex velocity potential is given by

$$
\phi_{2}=\sum_{n=0}^{\infty}\left[C_{3 n} R_{1 n}(\rho)+C_{4 n} R_{2 n}(\rho)\right] \cos n \theta
$$

Because the present investigation assumes the symmetric condition, the corresponding terms of the odd function $\sin n \theta$ are negligible.

The following boundary conditions are used for solving the problem:

$$
\frac{\partial \phi_{2}}{\partial \rho}=0 \quad \text { for } \rho=\rho_{1}\left(\text { or } r=r_{1}\right)
$$

Using (2.25), the relation between two coefficients $C_{3 n}$ and $C_{4 n}$ is given by

$$
C_{n}=\frac{C_{3 n}}{R_{2 n}^{\prime}\left(\rho_{1}\right)}=\frac{C_{4 n}}{R_{1 n}^{\prime}\left(\rho_{1}\right)} .
$$


The prime denotes the first derivative with respect to $\rho$. The rearrangement of (2.25) yields

$$
\phi_{2}=\sum_{n=0}^{\infty} C_{n}\left[R_{2 n}^{\prime}\left(\rho_{1}\right) R_{1 n}(\rho)+R_{1 n}^{\prime}\left(\rho_{1}\right) R_{2 n}(\rho)\right] \cos n \theta
$$

Two matching boundary conditions of continuity of flow mass and pressure are again adopted which are given by

$$
\begin{gathered}
\phi_{1}=\phi_{2} \quad \text { for } \rho=\rho_{0}, \\
\frac{\partial \phi_{1}}{\partial \rho}=\frac{\partial \phi_{2}}{\partial \rho} \quad \text { for } \rho=\rho_{0} .
\end{gathered}
$$

Substituting (2.29) into (2.23) and (2.25), we have

$$
\begin{aligned}
& C_{n} \Gamma_{n}=A_{I} \mathbf{i}^{n} \varepsilon_{n} J_{n}\left(\kappa_{0} \rho_{0}\right)+D_{n} \Psi_{n}\left(\rho_{0}\right), \\
& C_{n} \Lambda_{n}=A_{I} \mathbf{i}^{n} \varepsilon_{n} J_{n}^{\prime}\left(\kappa_{0} \rho_{0}\right)+D_{n} \Psi_{n}^{\prime}\left(\rho_{0}\right),
\end{aligned}
$$

where

$$
\begin{aligned}
& \Gamma_{n}=R_{2 n}^{\prime}\left(\rho_{1}\right) R_{1 n}\left(\rho_{0}\right)-R_{1 n}^{\prime}\left(\rho_{1}\right) R_{2 n}\left(\rho_{0}\right), \\
& \Lambda_{n}=R_{2 n}^{\prime}\left(\rho_{1}\right) R_{1 n}^{\prime}\left(\rho_{0}\right)+R_{1 n}^{\prime}\left(\rho_{1}\right) R_{2 n}^{\prime}\left(\rho_{0}\right) .
\end{aligned}
$$

The coefficients $C_{n}$ and $D_{n}$ are solved by (2.30) and are expressed by

$$
\begin{gathered}
C_{n}=-A_{I} \mathbf{i}^{n} \varepsilon_{n} \frac{\Psi_{n}\left(\rho_{0}\right) J_{n}^{\prime}\left(\kappa_{0} \rho_{0}\right)-\Psi_{n}^{\prime}\left(\rho_{0}\right) J_{n}\left(\kappa_{0} \rho_{0}\right)}{\Gamma_{n} \Psi_{n}^{\prime}\left(\rho_{0}\right)-\Lambda_{n} \Psi_{n}\left(\rho_{0}\right)}, \\
D_{n}=-A_{I} i^{n} \varepsilon_{n} \frac{\Gamma_{n} J_{n}^{\prime}\left(\kappa_{0} \rho_{0}\right)-\Lambda_{n} J_{n}\left(\kappa_{0} \rho_{0}\right)}{\Gamma_{n} \Psi_{n}^{\prime}\left(\rho_{0}\right)-\Lambda_{n} \Psi_{n}\left(\rho_{0}\right)} .
\end{gathered}
$$

\section{Results and Discussions}

In the following numerical results, we have typically set $\omega^{2} / g=1$, the inertial coefficient $S=1$ and $\bar{h}_{p}=\bar{h}_{w}=\bar{\xi}_{p}^{2}=\bar{\xi}_{w}^{2}=0.02$. The infinite series in the solution of the velocity potential should be truncated, indicating that the number of $N$ must be large enough to represent the infinity so that the infinite series in (2.23) and (2.28) can effectively accounts for the wave motion by a prescribed accuracy. For the case of $\alpha=1$ and $\alpha=2$, respectively. Figure 2 presents the variation of the relative wave runup height $\left(A / A_{I}\right)$ around the crosssection along the $y=0$. The result demonstrates the wave height distribution at different values of $\alpha$ for different permeable materials with variant porosities. In Figure 2(a), the wave height distribution before the island increases monotonically with the increase of $n_{0}$, but the variation of wave height distribution behind the island decreases monotonically with 


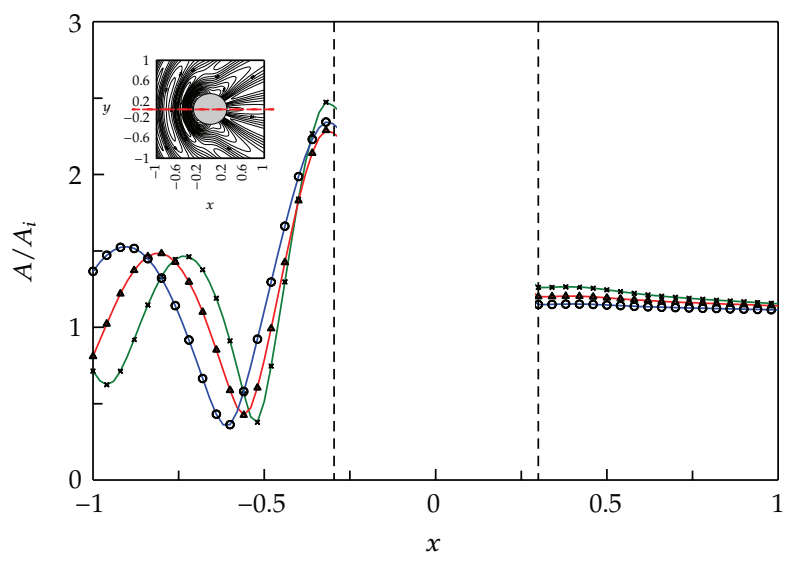

$$
\begin{aligned}
& \times n_{0}=0 \\
& \mathbf{\Delta} n_{0}=0.4 \\
& \ominus n_{0}=0.8
\end{aligned}
$$

(a) $\alpha=1$

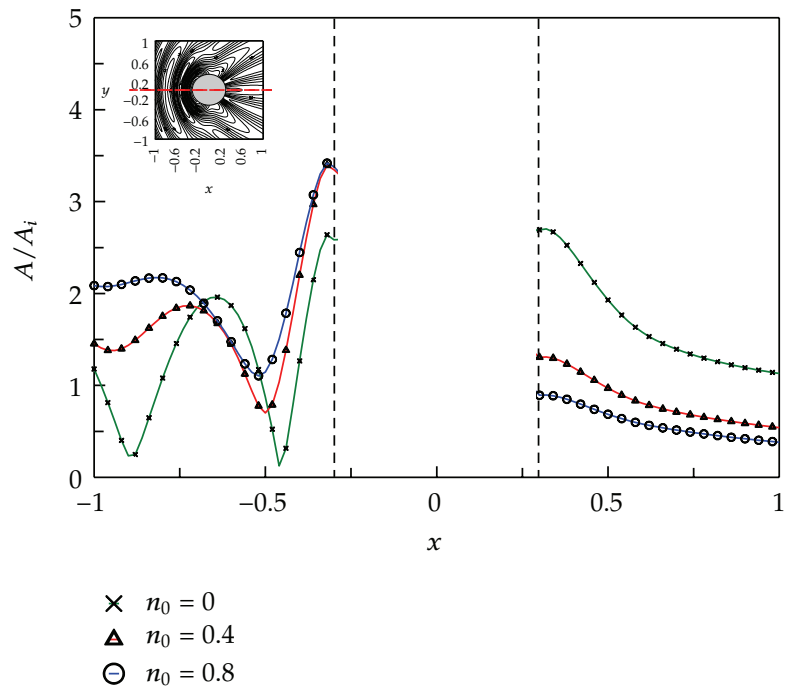

(b) $\alpha=2$

Figure 2: Effects of the porosity of the permeable material $\left(\rho_{0}=0.2 \pi, \rho_{1}=0.1 \pi, f_{p}=1\right)$.

the increasing $n_{0}$ for $\alpha=1, r_{0}=0.2 \pi$. Note that the variation of wave height distribution at $\alpha=2$ shows the same feature shown in Figure 2(b).

Figure 3 demonstrates the variation of wave height distribution at different values of $\alpha$ as a linearized friction coefficient changes. We notice that the wave height increases with the increase of the friction factor $f_{p}$ in the front of the island but opposite property behind the island.

Figures 4 and 5 show different bottom slopes for different values of $\alpha, \rho_{0}, \xi_{0}$ and $\rho_{1}$. It is interesting to note that a higher value could produce a steeper bottom geometry, 


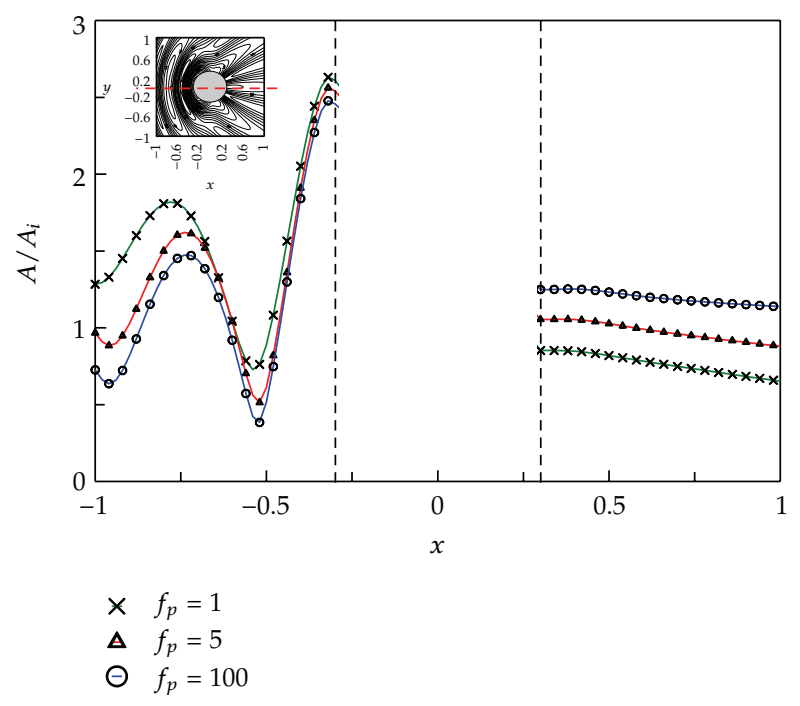

(a) $\alpha=1$

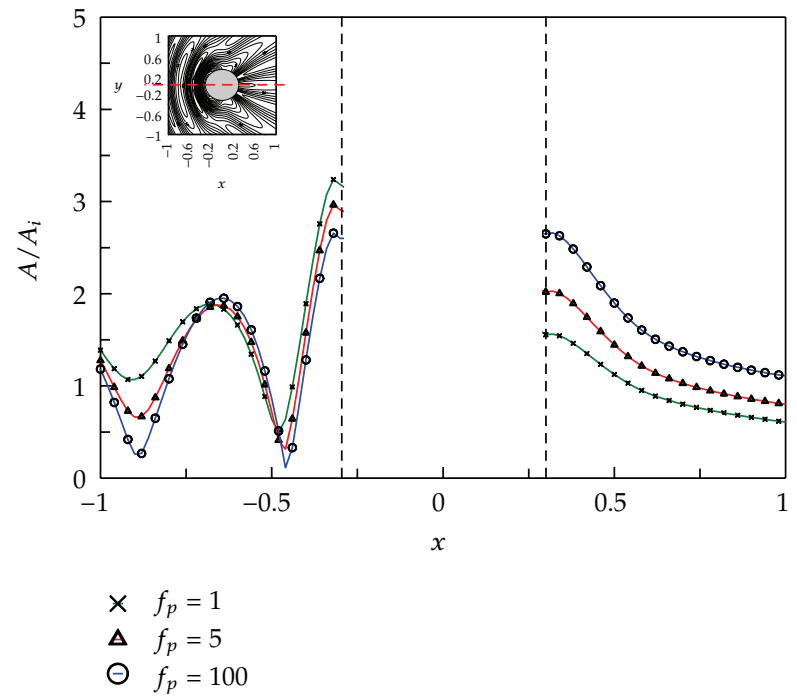

(b) $\alpha=2$

Figure 3: Effects of linearized friction coefficient $\left(\rho_{0}=0.2 \pi, \rho_{1}=0.1 \pi, n_{0}=0.5\right)$.

the intensity of wave ray focusing increases with the decrease of the bottom slope. Consequently, a significant increase of wave runup height is evident.

\section{Conclusions}

An analytic solution of the long-wave equation for waves propagating over a porous circular island was derived. The solution is in terms of the Bessel functions. The singularity was removed by the Frobenius series for the case of a parabolic island. The solution is able to account for the combined effect of refraction and diffraction around a circular island on the 


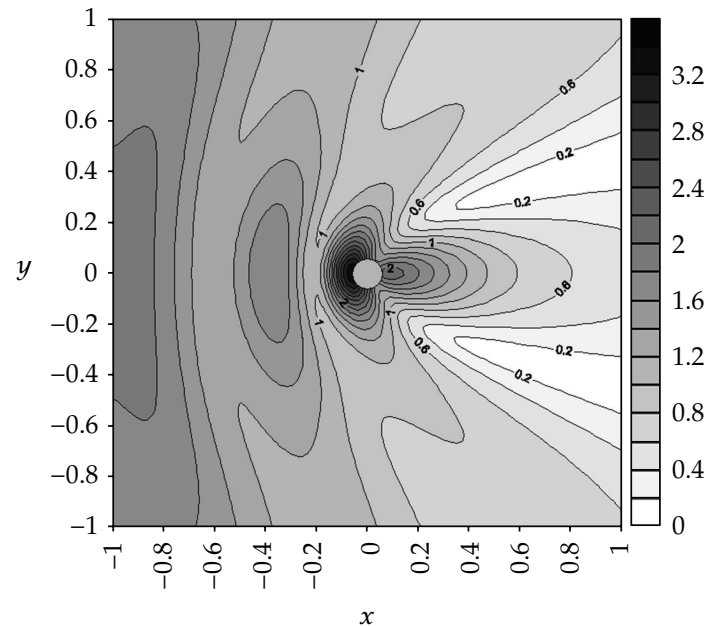

(a) $\rho_{1}=0.02 \pi$

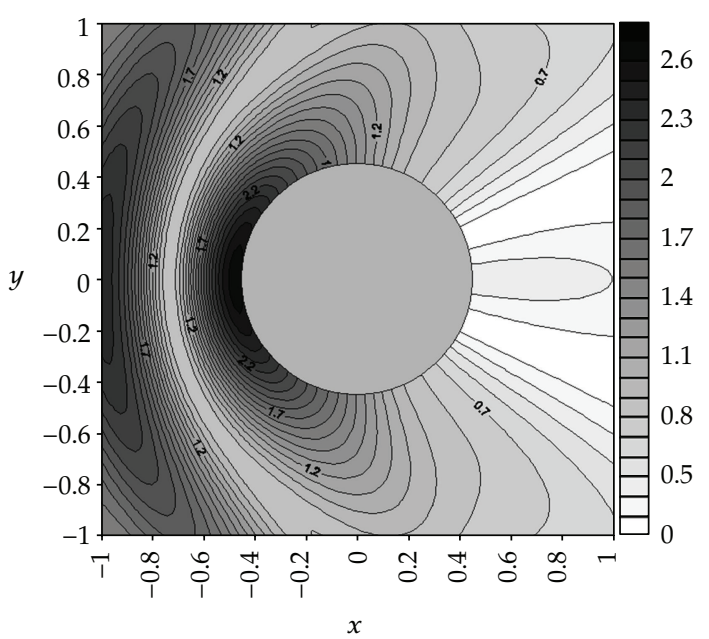

(b) $\rho_{1}=0.15 \pi$

Figure 4: Effects of island on wave scattering for $\alpha=1\left(\rho_{0}=0.2 \pi, f_{p}=1, n_{0}=0.5\right)$.

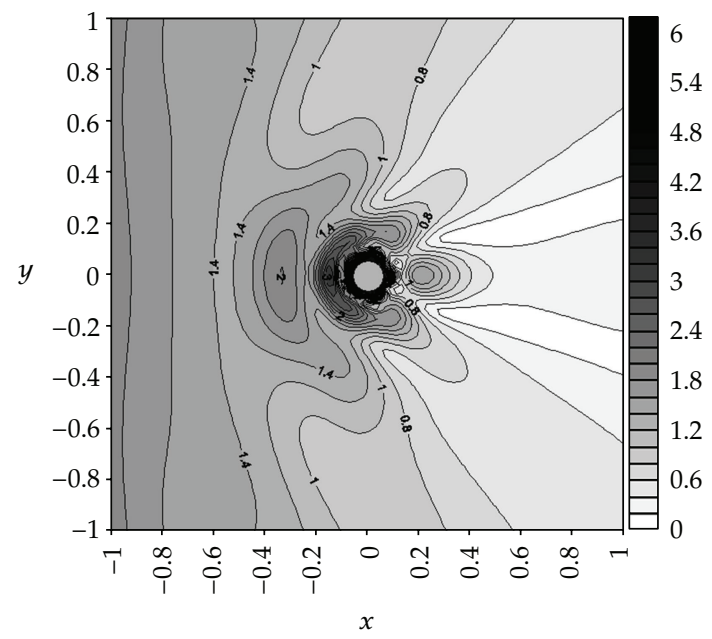

(a) $\rho_{1}=0.02 \pi$

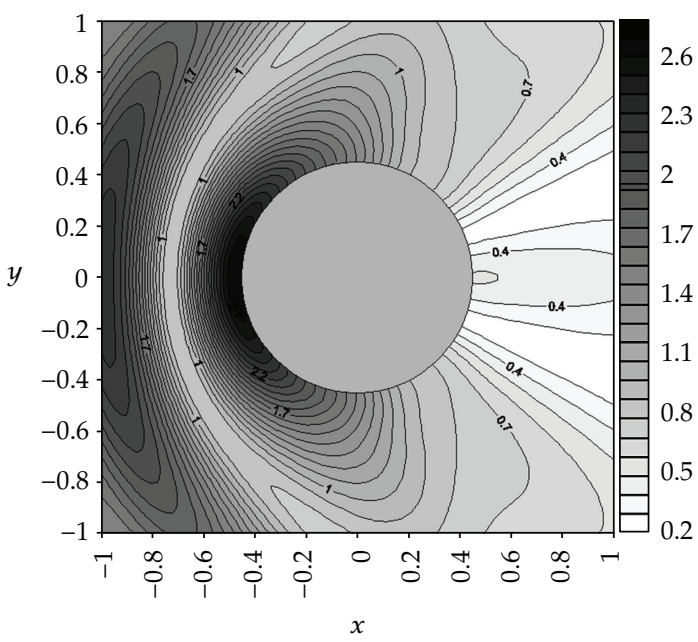

(b) $\rho_{1}=0.15 \pi$

Figure 5: Effects of island on wave scattering for $\alpha=2\left(\rho_{0}=0.2 \pi, f_{p}=1, n_{0}=0.5\right)$.

top of a shoal for arbitrary geometry described by two independent parameters. The effect of key parameters on waves scattering was investigated based on numerical calculations from the analytic solution.

\section{Appendix}

According to Rojanakamthorn et al. [19], the mild-slope equation for waves propagating over a permeable media is described by

$$
\nabla_{h}\left(I \nabla_{h} \phi\right)+k^{2} I \phi=0
$$


where

$$
I=\int_{-h_{w}}^{0} W^{2} d z+\frac{1}{\delta} \int_{-\left(h_{w}+h_{p}\right)}^{-h_{w}} P^{2} d z
$$

with

$$
\begin{aligned}
& W\left(z, h_{w}(x, y), h_{p}(x, y)\right) \\
& =\frac{(\delta-1) \sinh k\left[\left(h_{w}+h_{p}\right)+z\right] \tanh k h_{p}+\cosh k\left[\left(h_{w}+h_{p}\right)+z\right]\left(1-\delta \tanh ^{2} k h_{p}\right)}{(\delta-1) \sinh k\left(h_{w}+h_{p}\right) \tanh k h_{p}+\cosh k\left(h_{w}+h_{p}\right)\left(1-\delta \tanh ^{2} k h_{p}\right)} \\
& P\left(z, h_{w}(x, y), h_{p}(x, y)\right) \\
& =\frac{\delta \cosh k\left[\left(h_{w}+h_{p}\right)+z\right]}{\cosh ^{2} k h_{p}\left[(\delta-1) \sinh k\left(h_{w}+h_{p}\right) \tanh k h_{p}+\cosh k\left(h_{w}+h_{p}\right)\left(1-\delta \tanh ^{2} k h_{p}\right)\right]}
\end{aligned}
$$

being the depth functions in the water and porous layers respectively. The angular frequency $\omega$ is related to the wavenumber $k$ by the following dispersion relation:

$$
\frac{\omega^{2}}{g}=k \frac{(\delta-1) \sinh k\left(h_{w}-h_{p}\right)-(\delta+1) \sinh k\left(h_{w}+h_{p}\right)}{(\delta-1) \cosh k\left(h_{w}-h_{p}\right)-(\delta+1) \cosh \left(h_{w}+h_{p}\right)} .
$$

In order to consider the long wave limit, (A.3) should be expanded in polynomials of $k h_{w}$ and $k h_{p}$ as

$$
\begin{aligned}
& W\left(z, h_{w}(x, y), h_{p}(x, y)\right) \\
& \quad=(\delta-1)\left(k h_{p} \sinh k z\right)+\left[\cosh k z+k\left(h_{w}+h_{p}\right) \sinh k z\right]+O\left(k^{2} h_{w}^{2}, k^{2} h_{p}^{2}, k^{2} h_{w} h_{p}\right), \\
& P\left(z, h_{w}(x, y), h_{p}(x, y)\right)=\delta^{2}\left[\cosh k z+k\left(h_{w}+h_{p}\right) \sinh k z\right]+O\left(k^{2} h_{w}^{2}, k^{2} h_{p}^{2}, k^{2} h_{w} h_{p}\right) .
\end{aligned}
$$

Substituting (A.4) and (A.5) into (A.2) results in

$$
I=h_{w}+\delta h_{p}+O\left(k^{2} h_{w}^{2}, k^{2} h_{p}^{2}, k^{2} h_{w} h_{p}\right)
$$

Equations (A.1) and (A.7) give the long wave equation (2.1) of the present study. 
In addition, the dispersion relation (A.4) can also be expanded in polynomials of $k h_{w}$ and $k h_{p}$ as

$$
\frac{\omega^{2}}{g}=k\left(\delta k h_{p}+k h_{w}+O\left(k^{2} h_{w}^{2}, k^{2} h_{p}^{2}, k^{2} h_{w} h_{p}\right)\right)
$$

Equation (A.8) is sufficient to obtain the dispersion relation of long waves as given in (2.2).

\section{Acknowledgments}

This work was supported by the National Science Council of Taiwan, NSC 101-3113-P-006016 and Headquarters of University Advancement, National Cheng Kung University.

\section{References}

[1] J. C. W. Berkhoff, "Computation of combined refraction-diffraction," in Proceedings of the 13th International Conference on Coastal Engineering, pp. 471-490, ASCE, 1972.

[2] D. Porter and D. J. Staziker, "Extensions of the mild-slope equation," Journal of Fluid Mechanics, vol. 300, pp. 367-382, 1995.

[3] T. W. Hsu and C. C. Wen, "On radiation boundary conditions and wave transformation across the surfzone," China Ocean Engineering, vol. 15, no. 3, pp. 395-406, 2001.

[4] S. Homma, "On the behavior of seismic sea waves around circular island," Geophysical Magazine, vol. 21, pp. 199-209, 1950.

[5] A. C. Vastano and R. O. Reid, "Tsunami response for islands: verification of a numerical procedure," Journal of Marine Research, vol. 25, pp. 129-139, 1967.

[6] I. G. Jonsson, O. Skovgaard, and O. Brink-Kjaer, "Diffraction and refraction calculations for waves incident on an island," Journal of Marine Research, vol. 34, no. 3, pp. 469-496, 1976.

[7] K. Fujima and C. Goto, "Characteristics of long waves trapped by conical islands," Proceedings of the Japan Society of Civil Engineers, no. 497, pp. 101-110, 1994 (Japanese).

[8] K. Fujima, D. Yuliadi, C. Goto, K. Hayashi, and T. Shigemura, "Characteristics of long waves trapped by conical island," Coastal Engineering Journal, vol. 38, no. 2, pp. 111-132, 1995.

[9] Y. Zhang and S. Zhu, "New solutions for the propagation of long water waves over variable depth," Journal of Fluid Mechanics, vol. 278, pp. 391-406, 1994.

[10] S. Zhu and Y. Zhang, "Scattering of long waves around a circular island mounted on a conical shoal," Wave Motion, vol. 23, no. 4, pp. 353-362, 1996.

[11] T. H. Jung, C. Lee, and Y. S. Cho, "Analytical solutions for long waves over a circular island," Coastal Engineering, vol. 57, no. 4, pp. 440-446, 2010.

[12] X. Niu and X. Yu, "Long wave scattering by a vertical cylinder with idealized scour pit," Journal of Waterway, Port, Coastal and Ocean Engineering-ASCE, vol. 137, pp. 279-285, 2011.

[13] H. W. Liu, J. J. Xie, and Z. H. Luo, "An analytical solution for long-wave scattering by a circular island mounted on a general shoal," Journal of Waterway, Port, Coastal and Ocean Engineering-ASCE, vol. 139, no. 6, pp. 425-434, 2012.

[14] T. H. Jung and C. Lee, "Analytical solutions for long waves on a circular island with combined topographies," Wave Motion, vol. 49, pp. 152-164, 2012.

[15] H. W. Liu, P. Lin, and N. J. Shankar, "An analytical solution of the mild-slope equation for waves around a circular island on a paraboloidal shoal," Coastal Engineering, vol. 51, no. 5-6, pp. 421-437, 2004.

[16] X. Niu and X. Yu, "An analytic solution for combined wave diffraction and refraction around a vertical cylinder with idealized scour pit," Coastal Engineering, vol. 67, pp. 80-87, 2012.

[17] J. N. Hunt, "Direct solution of wave dispersion equation," Journal of Waterway, Port, Coastal and Ocean Engineering-ASCE, vol. 105, no. 1979, pp. 457-459, 1979. 
[18] X. Yu and B. Zhang, "An extended analytic solution for combined refraction and diffraction of long waves over circular shoals," Ocean Engineering, vol. 30, no. 10, pp. 1253-1267, 2003.

[19] S. Rojanakamthorn, M. Isobe, and A. Watanabe, "A mathematical model of wave transformation over a submerged breakwater," in Proceedings of the Coastal Engineering in Japan, vol. 32, pp. 209-234, 1989. 


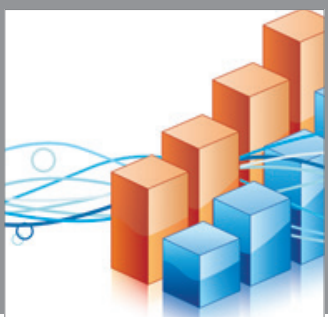

Advances in

Operations Research

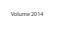

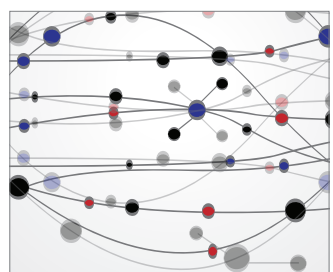

\section{The Scientific} World Journal
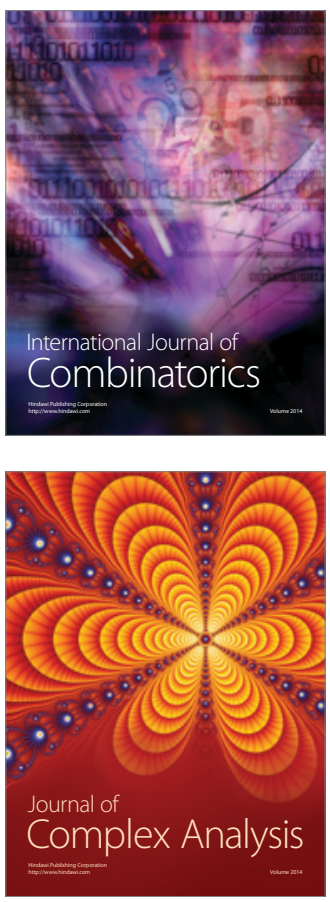

International Journal of

Mathematics and

Mathematical

Sciences
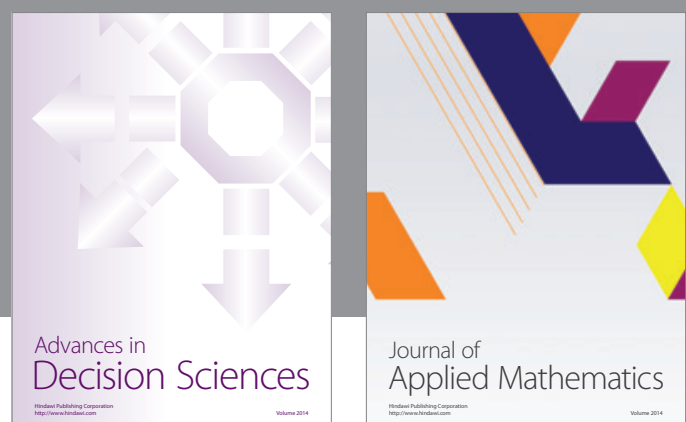

Journal of

Applied Mathematics
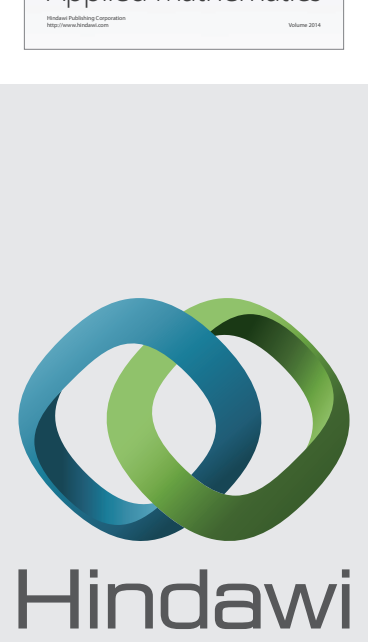

Submit your manuscripts at http://www.hindawi.com
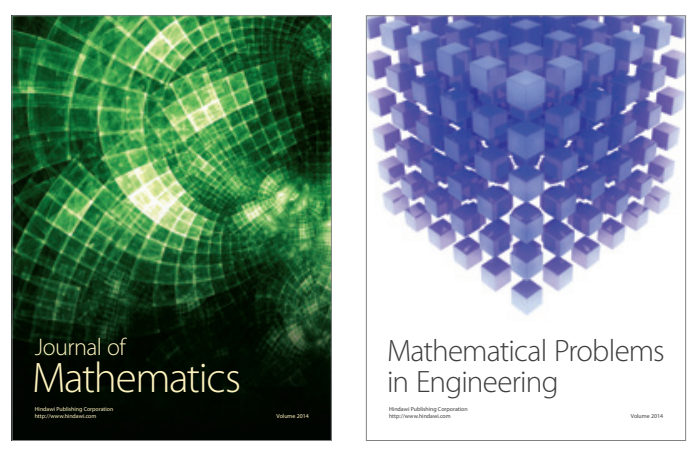

Mathematical Problems in Engineering
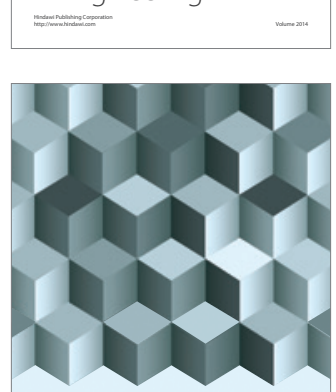

Journal of

Function Spaces
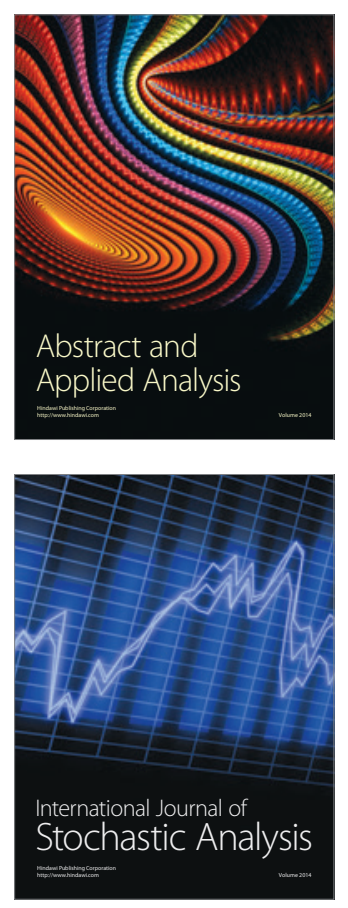

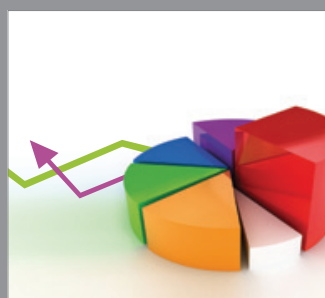

ournal of

Probability and Statistics

Promensencen
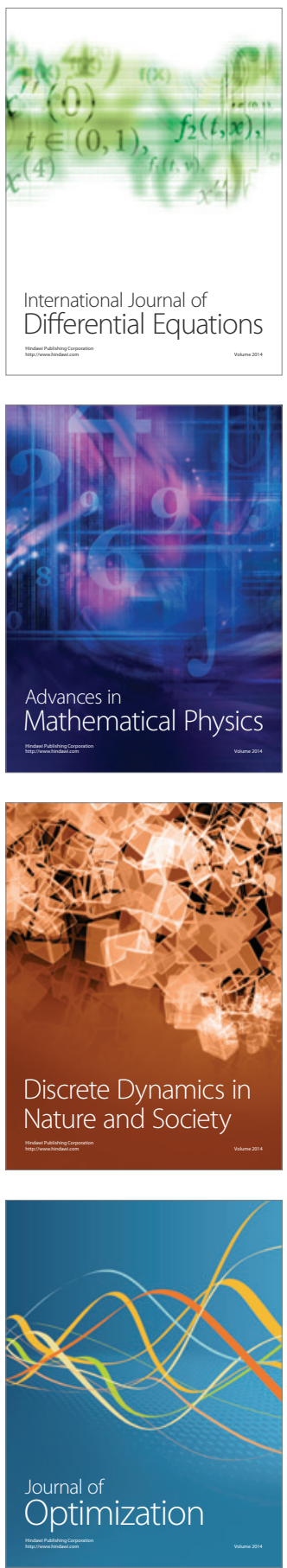